\title{
Neuromuscular conditions for physicians - what you need to know
}

\author{
Authors: Laura Edwards ${ }^{A}$ and Margaret Phillips ${ }^{B}$
}

The Royal College of Physicians (RCP) and the British Society of Rehabilitation Medicine co-hosted a meeting entitled 'Neuromuscular conditions for physicians - what you need to know' at the RCP on 30 November 2015. There was a series of talks, ranging from in-depth genetic and molecular descriptions of pathology to multidisciplinary management of chronic neuromuscular conditions, which stimulated lively debate and discussion. Some overarching themes emerged from the day, most notably: i) the changing expectations and survival rates in Duchenne muscular dystrophy (DMD), which are transforming this disorder into an adult as much as a paediatric condition; ii) the need for integrated management and good communication between services - whether primary, secondary and tertiary care, medical teams and intensivists, or the multiple teams involved in providing treatment to neuromuscular patients; and iii) in line with the above, the essential need for streamlining care such that patients can avoid spending most of their time attending outpatient appointments, and instead concentrate on living full lives and exploring educational, occupational, leisure and social opportunities.

\section{Background}

Neuromuscular disorders can range from inherited, lifelimiting conditions (eg Duchenne muscular dystrophy [DMD]) to acquired, often treatable and reversible disease (eg GuillainBarré syndrome). The majority of talks from the day focused on DMD and, in particular, on the changing diagnostic and therapeutic landscape that has led to incredible changes in survival, dramatically altering patient, family and healthcare team expectations.

DMD is the most common and disabling muscular dystrophy in the UK. ${ }^{1}$ It is an X-linked disorder, commonly diagnosed in boys around the age of 5 years when they present with marked physical impairment compared with their peers. They undergo progressive muscle deterioration and, without treatment, will require a wheelchair for mobility by the time they reach their teens. Death is typically seen without treatment before the age of 20 , commonly as a

Authors: ${ }^{\mathrm{A}}$ ACF ST5 rehabilitation medicine, UK; ${ }^{\mathrm{B}}$ consultant in rehabilitation medicine, Royal Derby Hospital, Derby, UK result of respiratory complications. ${ }^{2}$ Advances in treatment, notably the involvement of multidisciplinary teams, noninvasive ventilation and scoliosis surgery, have significantly increased the life expectancy of patients with DMD. ${ }^{2-4}$ More recently, boys with DMD have been routinely receiving steroid therapy, which reduces all-cause mortality and slows decline in function, ${ }^{2,5}$ and so it is likely that even more patients will continue to live longer lives.

\section{Genetics}

DMD is caused by mutations in the gene coding for dystrophin. Dystrophin is a membrane-stabilising protein (shock absorber), which links the intracellular cytoskeleton to the extracellular matrix. The common mutations in DMD either lead to frame shift, non-sense coding, and lack of production of protein, or the presence of an inappropriate stop mutation, leading to premature termination of translation by the ribosome and failure to form a functional protein. Lack of dystrophin production reduces the ability of skeletal and cardiac muscle cells to repair damage - even that caused by day-to-dayfunctioning - and results in weakness, wasting and fibrosis. Several different mutations occur in DMD and spontaneous mutations are relatively common; these can lead to challenges in antenatal diagnosis.

Postnatal genetic diagnosis can also be challenging. Although next generation sequencing and other advances have increased the accessibility to genetic screening and reduced the costs, genetic diagnosis should not and cannot be undertaken in isolation. Context is vital, in terms of clinical and family history and phenotype, and the results of muscle biopsy can also guide the diagnosis. Provision of a diagnosis to a patient and their family is not sufficient; it is important to be able to provide as much information as possible about prognosis, inheritance, therapeutic options, etc. Therefore, even at the first steps of investigation and treatment, input is required from paediatricians, geneticists, pathologists and geneticists.

\section{Genetic therapy}

Some exciting advances are being made in the realm of targeted genetic therapy, which is showing promising results in clinical trials. Work is ongoing to optimise these therapies and look at alternatives, including converting an out-of-frame deletion into an in-frame deletion by using anti-sense oligonucleotides. 
This would permit the production of some dystrophin and ameliorate the DMD phenotype so that it is similar to that of Becker muscular dystrophy, ${ }^{6}$ and enable the translating ribosome to bypass an inappropriate premature stop mutation, allowing the formation of a functional protein (eg ataluren).

Of course, the timing of such treatments is vital. Better results have been shown if treatment is started in patients who are ambulant - ie before too much muscle mass is lost. This really emphasises the importance of good basic early clinical care and coordination of therapy, so that patients can access such treatments and future trials with minimal disruption.

\section{Management approaches in DMD}

Reviews and recommendations regarding DMD management have been clearly outlined in the reviews by Bushby and colleagues. ${ }^{2,7}$ Some key principles were highlighted from the day's talks, however. DMD is a multi-organ and multi-specialty disease, involving many different services and care providers, some of which are mentioned in Box 1, although this list is in no way exhaustive and will vary depending on different patient and family needs. Some of these teams and individuals will be a constant throughout the patient journey; others might be involved for only a few weeks or months.

Regular reviews are important because several of the complications of DMD can be insidious. Left ventricular failure, for example, can be hard to spot in patients who are physically less active; symptoms tend not to arise until left ventricular ejection fraction is below $20 \%$, by which time a large amount of damage has been done and there is less of a window for therapies - including steroids, angiotensinconverting enzyme inhibitors, eplerenone or even physical interventions such as pacemakers or internal defibrillators - to be effective. ${ }^{5,7,8}$

Doctors also need to discuss patients' expectations and wishes in a timely manner. For instance, knowing in advance whether someone would want a tracheostomy and long-term ventilation makes decision making about admission to the adult intensive care unit and treatment, while never easy, hopefully less traumatic. In such a setting, experienced healthcare professionals might also need to educate their colleagues on the changes in life expectancy in DMD, to try to avoid or reduce therapeutic nihilism.

It easy to see how contact with so many services on a regular basis could lead to duplication of investigations or interventions and -particularly with services spread across departments, hospitals and sometimes even cities or regions - how a huge amount of patients' lives can be spent attending outpatient appointments, only to find that each specialist might not have the relevant information, necessitating the relating of lengthy histories each time.

Arguments can be made for the one-stop-shop clinic, where patients are seen at a single appointment by multiple specialists, but this approach might not be as successful in less urban areas and can be intensive for patients. It is clear that there is a need for a coordinator of care, who can liaise with the different specialties at appropriate times, ensure that screening and routine investigations are carried out as required, and perhaps act as a first, consistent point of contact for patients and families. It has been suggested that rehabilitation physicians could be ideally placed for such a role,
Box 1. Involvement of different specialists and

providers in the care of patients with Duchenne

muscular dystrophy.

\section{Medical}

Paediatric clinics

Transition clinics

Adult clinics

Genetics

Neurology

Pathology

Neuromuscular disease

Rehabilitation

Orthopaedics

Cardiology

Respiratory

Pain management

Psychiatry

Endocrinology

Anaesthesia

Intensive care medicine

Allied healthcare professionals

Musculoskeletal physiotherapy

Occupational therapy

Speech and language therapy

Dietetics

Psychology

Social work

Care coordinator

Technical staff (electrocardiography, lung function etc)

Phlebotomy

Neurological physiotherapy

Non-invasive ventilation team

Respiratory physiotherapy

Play therapy

Nursing

Family support

Administrative staff

because they are experienced in dealing with patients with long-term neurological conditions of uncertain outcome and liaising between different specialties. They are also familiar with holistic care within the International Classification of Functioning, Disability and Health framework and, equally importantly, if not more so, aim to minimise the impact of disabilities so that patients can lead as normal and independent a life as possible. Guidelines are currently being developed to clarify the role of the rehabilitation physician in neuromuscular conditions. 


\section{Summary}

$>$ Anticipatory and preventive management of DMD is key. National and international guidelines could help with implementation. $^{2}$

$>$ Novel interventions, such as those being developed in surgery, genetics and cardiology, cannot make up for ground lost by poor medical care early on. The need for integrated, coordinated management has never been more important.

> The changing outcomes in DMD must lead to re-evaluation of expectations from patients, families and healthcare professionals.

\section{References}

1 Norwood F, Harling C, Chinnery P et al. Prevalence of genetic muscle disease in Northern England: in-depth analysis of a muscle clinic population. Brain 2009;132:3175-86.

2 Bushby K, Finkel R, Birnkkrant D et al. Diagnosis and management of Duchenne muscular dysytrophy, part 1: diagnosis, and pharmacological and psychosocial management. Lancet Neurol 2010;9:77-93.
3 Eagle M, Baudouin S, Chandler C et al. Survival in Duchenne muscular dystrophy: improvements in life expectancy since 1967 and the impact of home nocturnal ventilation. Neuromusc Disord 2002;12:926-9.

4 Eagle M, Bourke J, Bullock R et al. Managing Duchenne muscular dystrophy - the additive effect of spinal surgery and home nocturnal ventilation in improving survival. Neuromusc Disord 2007;17:470-5.

5 Schram G, Fournier A, Leduc $\mathrm{H}$ et al. All-cause mortality and cardiovascular outcomes with prophylactic steroid therapy in Duchenne muscular dystrophy. J Am Coll Cardiol 2013;61:948-54.

6 Kole R, Krieg A. Exon skipping therapy for Duchenne muscular dystrophy. Adv Drug Deliv Rev 2015;87;104-7.

7 Bushby K, Finkel R, Birnkkrant D et al. Diagnosis and management of Duchenne muscular dysytrophy, part 2: implementation of multidisciplinary care. Lancet Neurol 2010;9:177-89.

8 Raman S, Hor K, Mazur W et al. Eplerenone for early cardiomyopathy in Duchenne muscular dystrophy: a randomised, doubleblind, placebo-controlled trial. Lancet Neurol 2015;14:153-61.

Address for correspondence: Dr L Edwards, Division of Rehabilitation Medicine, Royal Derby Hospital, Uttoxeter Road, Derby DE22 3NE, UK.

Email: ljedwards@doctors.net.uk
'This landmark report lays out in the starkest terms yet the devastating impact air pollution is having on our health, our economy and our society as a whole.'

\section{Every breath we take The lifelong impact of air pollution}

This major report plainly sets out the dangerous impact that air pollution has on our nation's health. Compiled by experts in medicine and environmental sciences, the report discusses the current evidence and draws up recommendations for action.

ISBN 978-1-86016-567-2 £15 including $p+p$ or free to download

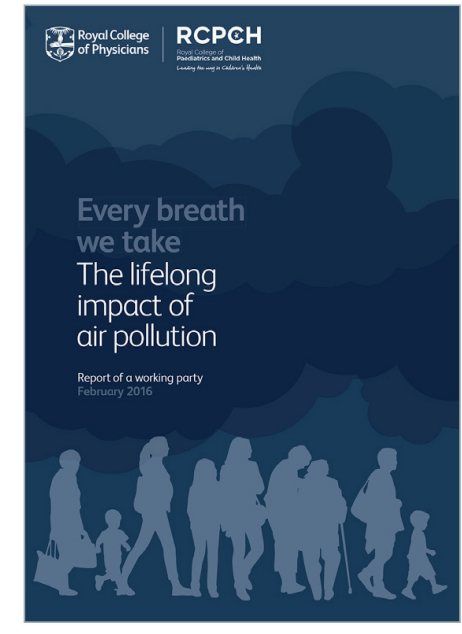

Order a copy: shop.rcplondon.ac.uk
Download the report: rcplondon.ac.uk/pollution

\section{Royal College of Physicians}

